\title{
Avaliação de Bem-Estar Psicológico e Sintomas Depressivos em Idosos
}

\author{
Saudáveis \\ Daiane Santos de Oliveira* \\ Pontifícia Universidade Católica do Rio Grande do Sul - PUC-RS, Porto Alegre, RS, Brasil \\ ORCID: https://orcid.org/0000-0002-5116-4393 \\ Manuela Polidoro Lima** \\ Pontifícia Universidade Católica do Rio Grande do Sul - PUC-RS, Porto Alegre, RS, Brasil \\ ORCID: https://orcid.org/0000-0002-9366-7829 \\ Cleber Gibbon Ratto*** \\ Centro Universitário Metodista - IPA, Porto Alegre, RS, Brasil \\ ORCID: https://orcid.org/0000-0002-9059-728X \\ Tainá Rossi***** \\ Faculdade Meridional - IMED, Passo Fundo, RS, Brasil \\ ORCID: https://orcid.org/0000-0002-4131-4247 \\ Rafael Reiman Baptista***** \\ Pontifícia Universidade Católica do Rio Grande do Sul - PUC-RS, Porto Alegre, RS, Brasil \\ ORCID: https://orcid.org/0000-0003-1937-6393 \\ Tatiana Quarti Irigaray****** \\ Pontifícia Universidade Católica do Rio Grande do Sul - PUC-RS, Porto Alegre, RS, Brasil \\ ORCID: https://orcid.org/0000-0002-3078-4219
}

\section{RESUMO}

O objetivo deste trabalho foi avaliar a relação entre bem-estar psicológico e a presença de sintomas depressivos em idosos saudáveis. Os instrumentos utilizados foram: ficha de dados sociodemográficos, EDEP e BDI-II. Os dados foram analisados com o auxílio do SPSS, por meio de frequências, média, desvio padrão e correlações de Spearman. A amostra foi composta por 64 idosos, com média de idade de 69,61 (DP=7,58) e média de escolaridade de 4,59 anos de estudo $(\mathrm{DP}=2,61)$. Os resultados indicaram correlação inversa, moderada e significativa entre sintomatologia depressiva e os domínios autonomia, ambiente e relações positivas com os outros, encontrou-se ainda correlação inversa e fraca entre sintomatologia depressiva e crescimento pessoal. Acredita-se que escolaridade e atividade física podem ser fatores protetivos para depressão e bem-estar psicológico no envelhecimento. Por fim, levanta-se a hipótese de haver uma retroalimentação entre as variáveis que se correlacionaram entre si de forma significativa neste estudo.

Palavras-chave: envelhecimento, idoso, depressão. 


\title{
Evaluation of Psychological Well-Being and Depressive Symptoms in
}

\author{
Healthy Elderly People
}

\begin{abstract}
The main objective of this study was to evaluate the relationship between psychological wellbeing and depressive symptoms in healthy elderly people. The instruments used were: sociodemographic data questionnaire, EDEP and BDI-II. The data were analyzed in the SPSS using mean and SD, and Spearman correlations. The sample consisted of 64 elderly, with a mean age of 69.61 years $(\mathrm{SD}=7.58)$ and a mean educational level of 4.59 years $(\mathrm{SD}=2.61)$. The results indicate an inverse, moderate and significant correlation between depressive symptomatology and the domains: autonomy, environment, and positive relationships with others. There was also an inverse and weak correlation between depressive symptomatology and personal growth domain. It is believed that education level can be a protective factor for depression and psychological well-being in aging. Finally, the hypothesis is that there is a feedback cycle between the significantly correlated variables in this study.
\end{abstract}

Keywords: aging, elderly, depressive symptomatology.

\section{Evaluación de Bienestar Psicológico y Sintomas Depresivos en Los Idosos}

\author{
Saludables
}

\section{RESUMEN}

El objetivo de este trabajo fue evaluar la relación entre bienestar psicológico y la presencia de síntomas depresivos en ancianos sanos. Los instrumentos utilizados fueron: ficha de datos sociodemográficos, EDEP y BDI-II. Los datos fueron analizados con la ayuda del SPSS, por medio de frecuencias, media, desviación estándar y correlaciones de Spearman. La muestra fue compuesta por 64 ancianos, siendo la media de edad fue 69,61 $(+7,58)$ y la media de escolaridad fue de 4,59 años de estudio $(+2,61)$. Los resultados indicaron correlación inversa, moderada y significativa entre sintomatología depresiva y los dominios autonomía, ambiente y relaciones positivas con los demás, se encontró una correlación inversa y débil entre sintomatología depresiva y crecimiento personal. Se cree que la escolaridad y la actividad física son factores protectores para la depresión y el bienestar psicológico en el envejecimiento. Por último, se plantea la hipótesis de que hay una retroalimentación entre las variables que se correlacionaron entre sí de forma significativa en este estudio.

Palabras clave: envejecimiento, personas de edad avanzada, la depresión.

O processo de envelhecimento humano é permeado por diversas mudanças físicas, psicossociais e psicológicas, como o declínio das funções executivas, responsáveis pela linguagem, memória e aprendizagem (Tsai et al., 2016). Além disso, algumas alterações 
multifatoriais (viuvez, redução de mobilidade, condições clínicas como hipertensão e diabetes) podem afetar os hábitos adquiridos ao longo da vida. Entre elas a aposentadoria, capaz de provocar uma ruptura na rotina ativa de trabalho do idoso, gerando assim, desconforto e sensação de incapacidade. Pode ocorrer também alterações de papéis, como por exemplo, o idoso que ocupava um papel de cuidador, agora adquire o papel de alguém a ser cuidado (Silva et al., 2017).

Estudos apontam que a população idosa está entre as mais vulneráveis para o desenvolvimento de patologias, há evidências de que a depressão é a psicopatologia com maior incidência nesta população, afetando diretamente a qualidade de vida (Lippert, Fernandes, Jerônimo, \& Gomes, 2017; Ramos, Carneiro, Barbosa, Mendonça, \& Caldeira, 2015). Desta forma, aumenta também o risco de mortalidade na população idosa pois torna mais suscetível a presença de doenças físicas altamente incapacitantes, como a diabetes por exemplo, e mais vulnerável ao isolamento social (Silva et al., 2017).

Além disso, a depressão pode ser entendida como um processo de desvalorização, sentido pelo sujeito perante sua vida, impossibilitando a construção de novas perspectivas sobre o futuro (Kehl, 2015). Assim, a depressão pode acarretar uma redução no bem-estar psicológico dificultando as condições para a realização de mudanças positivas (Ramos et al., 2019; Silva, 2010).

O conceito de bem-estar psicológico encontra-se dentro da perspectiva eudaimônica, na qual evidencia-se uma felicidade mais duradoura, relacionada a um propósito de vida, à autorrealização e a potencialidades (Fave, Brdar, Freire, Vella-Brodrick, \& Wissing, 2011; Ryff \& Singer, 2008). De acordo com Ryff (2014), há seis dimensões estruturais do funcionamento psicológico: a) propósito de vida: capacidade de dar sentido, propósito a sua vida, criação de objetivos; b) autonomia: capacidade para seguir as próprias opiniões e convicções próprias; c) crescimento pessoal: abertura a novas experiências, potencialidade e autorrealização; d) domínio sobre o ambiente: administração dos ambientes nos quais o indivíduo está inserido, utilizando seu potencial; e) relações positivas: relações de afeto, criação de vínculo com o outro; e f) autoaceitação: autoconhecimento e aceitação de si.

Foi criado a partir do conceito de Erikson (1998) a dimensão geratividade que se refere a precaução e instigação com a continuidade das futuras gerações, incluindo procriatividade, produtividade e criatividade. Deste modo, é explicado a partir da necessidade interna de deixar um legado, repassar seu conhecimento e experiências para a próxima geração (Queroz \& Neri, 2005). 
O bem-estar psicológico regula o sentimento de satisfação com a vida e o crescimento pessoal, e não declina com o passar do tempo. Porém, pode ser afetado pelas dificuldades e limitações vivenciadas, sejam elas cognitivas, físicas ou psicológicas (Resende \& Neri, 2009; Warr, 2011).

Segundo Irigaray, Schneider e Gomes (2011), a interação social dos idosos pode promover um efeito positivo na redução do sentimento de solidão e diminuição do impacto de sentimentos negativos perante a vida. Esses resultados foram encontrados a partir da investigação dos efeitos de um treino cognitivo no bem-estar psicológico e na qualidade de vida de idosos divididos em dois grupos (controle e experimental). Este estudo utilizou os instrumentos WHOQOL - bref, e Escala de Desenvolvimento Pessoal (EDEP) e concluiu que a prática de exercícios físicos, leitura, e atividades culturais podem aumentar o bem-estar psicológico dos idosos, possibilitando a interação social, a promoção de saúde, e a expectativa de vida (Irigaray et al., 2011).

Portanto, o bem-estar psicológico é fundamental para a promoção de saúde na velhice (Tallmann, Lenardt, Kletemberg, Michel, \& Lourenço, 2013), uma vez que este processo pode ser caracterizado por diferentes patologias. A depressão é uma das doenças mais frequentemente diagnosticadas em idosos, e está associada à baixa satisfação com a vida. Sendo assim, este trabalho tem como objetivo avaliar a relação entre bem-estar psicológico e a presença de sintomas depressivos em idosos saudáveis, visto que, no processo demencial apresentam-se sintomas semelhantes à depressão.

\section{Método}

Esta pesquisa quantitativa exploratória é um recorte de um projeto guarda-chuva denominado "Capacidade Funcional e sua relação com Aspectos Psicológicos e Cognitivos de idosos", desenvolvido por meio da parceria entre dois grupos de pesquisa de uma Universidade Brasileira, sendo um deles da Psicologia e o outro da Educação Física. A parceria nasceu da ideia inicial de acompanhar e avaliar aspectos físicos (motores) e psicológicos de idosos que praticavam atividade física regular. Os Projetos Guarda-Chuva se articulam e/ou se desdobram em outros (sub)projetos que são desenvolvidos em parceria. 
Daiane Santos de Oliveira, Manuela Polidoro Lima, Cleber Gibbon Ratto, Tainá Rossi, Rafael Reiman Baptista, Tatiana Quarti Irigaray

\section{Participantes}

A amostra foi composta por 64 idosos selecionados por conveniência, participantes de um projeto de extensão sobre atividade física e envelhecimento. Entre eles, 58 (90,6\%) eram do sexo feminino e seis $(9,4 \%)$ do sexo masculino. A média de idade dos participantes foi 69,61 anos $(\mathrm{DP}=7,58)$ e de escolaridade foi 4,59 anos ( $\mathrm{DP}=2,61)$. Além disso, 32,8\% eram casados, $37,5 \%$ eram viúvos e 15,6\% solteiros. Ademais, 67,2\% eram aposentados, 18,8\% pensionista, $12,5 \%$ estavam trabalhando, e 1,6\% nunca haviam trabalhado. Destes idosos, $39,1 \%$ moravam sozinhos, $23,4 \%$ com filhos e os demais com outros membros da família como netos, cônjuge, mãe, entre outros. Os critérios de inclusão abarcaram: 1) ter idade igual ou maior que 60 anos; 2) não possuir diagnóstico de doença neurológica (autorrelato); e 3) ter boa capacidade funcional (avaliado por educadores físicos). Utilizou-se como critério de exclusão: 1) os que não concluíram a bateria de instrumentos.

\section{Instrumentos}

Ficha de dados sociodemográficos. Coletou informações sobre idade, sexo, estado civil, escolaridade, situação de moradia, profissão exercida, ocupação atual, atividades de lazer, entre outras;

Escala de Desenvolvimento Pessoal (EDEP, Neri, 1999). Instrumento composto por 30 itens, destes, 18 foram elaborados a partir de seis dimensões psicológicas:1) autonomia; 2) propósito de vida; 3) domínio do ambiente; 4) crescimento pessoal; 5) auto aceitação; e 6) relações positivas com os outros (três itens para cada dimensão definida pela autora) (Ryff, 1989; Ryff \& Keyes, 1995); e 12 itens acrescentando o conceito de geratividade e seus componentes (criar, manter e oferecer) (Resende \& Neri, 2009). Cada item é avaliado por uma escala tipo Likert, contendo cinco pontos (1- pouquíssimo; 2- pouco; 3- mais ou menos; 4-muito; 5- muitíssimo). A escala apresentou um alfa de Cronbach 0,90 (Cachioni et al., 2017) e de 0,901 (Resende \& Neri, 2009) em estudos anteriores.

Inventário Beck de Depressão-II - BDI-II (Beck, Steer, \& Brown, 1996; adaptado por Gorenstein, Pang, Argimon, \& Werlang, 2011). Instrumento de auto aplicação, composto por 21 itens, que se propõe a mensurar a intensidade de sintomatologia depressiva. O manual sugere pontos de corte para avaliação da intensidade dos sintomas depressivos, sendo eles: mínimo (0-13), leve (14-19), moderado (20-28) e grave (29-63). Este instrumento apresentou um alfa de Cronbach 0,91 (Silva, Wendt, \& Argimon, 2018). 


\section{Procedimentos}

Coleta de dados. Os idosos foram convidados a participar do estudo por contato telefônico, a partir de uma lista de participantes do projeto de extensão. Para aqueles que aceitaram, agendou-se horário prévio conforme a disponibilidade de participação. Previamente ao início da coleta de dados, os participantes preencheram e assinaram o Termo de Consentimento Livre e Esclarecido (TCLE) concordando com sua voluntariedade. A coleta de dados foi realizada em grupos de até 10 participantes, em salas de aula da própria instituição universitária que desenvolveu a pesquisa, com duração aproximada de uma hora.

Os participantes foram avaliados por psicólogos e alunos de iniciação científica do curso de Psicologia, que receberam treinamento prévio à aplicação dos instrumentos. Aos participantes do estudo foi garantido sigilo quanto a sua identidade, sendo a participação voluntária. Os participantes que mostraram necessidade de acompanhamento psicológico durante a coleta de dados foram encaminhados aos serviços especializados disponíveis na comunidade.

Análise de dados. Os dados foram organizados e analisados com o auxílio do software estatístico Statistical Package for the Social Sciences (SPSS) versão 17.0 para Windows, considerando resultados significativos aqueles com $p \leq 0,05$. A descrição dos dados foi realizada por meio de frequências absolutas (n), relativas (\%), por média e desvio padrão. Correlações de Spearman foram utilizadas para verificar a relação entre as variáveis, de acordo com o resultado do Teste Kolmogorov-Smirnov, que indicou distribuição não normal dos dados. Para avaliar a relação de linearidade, foi estimado o coeficiente de correlação de Pearson, conforme a classificação de Cohen (1988): fraco quando o valor for menor que 0,3 ; moderado entre 0,3 e 0,6 ; forte de 0,6 a 0,8 ; e muito forte acima de 0,9 .

\section{Procedimentos éticos}

O presente estudo é recorte de um projeto maior denominado "Capacidade Funcional e sua relação com Aspectos Psicológicos e Cognitivos de idosos", submetido e aprovado por uma Comissão Científica e por um Comitê de Ética, reconhecido pelo Conselho Nacional de Saúde sob o CAAE: 55674116.3.0000.5336. 
Daiane Santos de Oliveira, Manuela Polidoro Lima, Cleber Gibbon Ratto, Tainá Rossi, Rafael Reiman Baptista, Tatiana Quarti Irigaray

\section{Resultados}

A amostra total foi composta por 64 idosos saudáveis, com média de idade de 69,61 anos $(D P=7,58)$ e média de escolaridade de 4,59 anos de estudo $(D P=2,61)$. Outras características sociodemográficas da amostra apresentam-se na Tabela 1, conforme segue.

Tabela 1

Informações Sociodemográficas dos Participantes

\begin{tabular}{lcc}
\hline Variáveis & $M$ & $D P$ \\
\hline Idade & 69,61 & 7,58 \\
Escolaridade (anos) & 4,59 & 2,61 \\
\cline { 2 - 3 } Sexo & $N$ & $\%$ \\
\cline { 2 - 3 } Feminino & 58 & 90,6 \\
$\quad$ Masculino & 6 & 9,4 \\
Estado Civil & & \\
Viúvo & 24 & 37,5 \\
Casado & 21 & 32,8 \\
Solteiro & 10 & 15,6 \\
Divorciado & 9 & 14,1 \\
Ocupação Atual & & \\
Aposentado & 43 & 67,2 \\
Pensionista & 12 & 18,8 \\
Trabalhando & 8 & 12,5 \\
Nunca trabalhou & 1 & 1,5 \\
Com quem mora & & \\
Sozinho & 25 & 39,1 \\
Cônjuge & 16 & 25 \\
Filhos & 19 & 29,7 \\
Outros & 4 & 6,2 \\
\hline & &
\end{tabular}

Encontrou-se a prevalência de mulheres na amostra, viúvas, já aposentadas e que residem sozinhas. A seguir, a Tabela 2 apresenta a média de classificação da Escala de BemEstar Psicológico (EDEP) aplicada em idosos saudáveis. 
Daiane Santos de Oliveira, Manuela Polidoro Lima, Cleber Gibbon Ratto, Tainá Rossi,

Tabela 2

Média de classificação da Escala de Bem-Estar Psicológico (EDEP)

\begin{tabular}{lcc}
\hline Variáveis & $M$ & $D P$ \\
\hline Bem-Estar & 72,22 & 17,26 \\
Autoaceitação & 68,36 & 16,41 \\
Autonomia & 74,05 & 16,6 \\
Crescimento Pessoal & 74,04 & 18,94 \\
Domínio sobre o Ambiente & 75,28 & 14,47 \\
Propósito de Vida & 67,46 & 18,19 \\
Relação Positiva com os Outros & & \\
Geratividade & 57,6 & 19,04 \\
Criar & 64,86 & 14,35 \\
Oferecer & 63,35 & 15,08 \\
Manter & & \\
\hline
\end{tabular}

Nota. Média 0-100

O nível de bem-estar encontrado obteve média de $104,73(D P=14,25)$ pontos, sendo a pontuação mínima atingida igual a 74 e a máxima igual 145 pontos. A Tabela 3 apresenta os resultados obtidos do Inventário Beck de Depressão-II (BDI-II).

Tabela 3

Classificação BDI-II

\begin{tabular}{lcc}
\hline & $N$ & $\%$ \\
\hline Mínimo 0-13 & 45 & 70,3 \\
Leve 14-19 & 11 & 17,2 \\
Moderado 20-28 & 3 & 4,7 \\
Grave 29-63 & 5 & 7,8 \\
\hline
\end{tabular}

O resultado aponta a presença de sintomatologia depressiva nos participantes do estudo. Encontra-se na Tabela 4 o resultado da correlação realizada entre sintomas depressivos e os domínios da EDEP.

Tabela 4

Correlação entre EDEP e BDI-II

\begin{tabular}{lcc}
\hline & $r$ & $p$ \\
\hline Autonomia & $-0,35$ & 0,005 \\
Domínio sobre o Ambiente & $-0,41$ & 0,001 \\
Relação Positiva com os Outros & $-0,38$ & 0,002 \\
Crescimento Pessoal & $-0,28$ & 0,025
\end{tabular}

Nota. ${ }^{*} p<0,05$. **indice $\mathrm{R}=0,00$ bem fraca; 0,20 fraca; 0,40 moderada; 0,70 forte e 0,90 muito forte 
As correlações entre sintomas depressivos e os domínios auto aceitação, propósito de vida e geratividade da EDEP não obtiveram resultados estatisticamente significativos. Ademais, não foram encontradas correlações estatísticas significativas entre escolaridade e depressão.

\section{Discussão}

O objetivo deste estudo foi avaliar a relação entre bem-estar psicológico e a presença de sintomas depressivos em idosos saudáveis. Os resultados evidenciaram que quanto maior a sintomatologia depressiva, menor o bem estar psicológico - especificamente o bem estar referente aos domínios de ambiente, autonomia, relações positivas com os outros e crescimento pessoal. De forma geral, quanto maior a presença de sintomas depressivos menor o bem-estar psicológico dos idosos. Os achados deste estudo corroboram com a pesquisa de Andrade, Duarte, Cruz, Alburquerque e Chaves (2019), que em seus resultados obteve índices menores de depressão e isolamento social em idosos com maior repertório de habilidades emocionais, as quais potencializam a segurança, autonomia e relações com os outros.

O domínio ambiente compreende o controle do indivíduo nas atividades complexas da vida pessoal, familiar e atividade profissional, de modo a sentir-se satisfeito. No questionário EDEP corresponde às questões "2) Eu me sinto dono (a) da minha própria vida"; "3) Eu consigo enfrentar com vigor e coragem os problemas do dia-a-dia" e "10) Eu consigo dar conta de várias responsabilidades da minha vida cotidiana”. Segundo Irigaray et al., (2011), idosos com senso de competência tendem a manter-se mais ativos e independentes, exercendo atividades nas quais possam controlar o próprio ambiente. O estudo de Cachioni et al., (2017) realizado com idosos que frequentavam a Universidade Aberta a Terceira Idade (UnATI), indicou que os contatos sociais proporcionados pela instituição de ensino superior atuaram como suporte emocional. Portanto, as redes de suporte social são essenciais no processo de envelhecimento, auxiliando no sentimento de pertencimento e fortalecendo vínculos de amizades.

Os achados deste trabalho indicaram que $12,5 \%$ dos idosos participantes da pesquisa trabalhavam. O estudo de Silva (2017) analisou a população idosa ativa laboralmente e a sintomatologia depressiva, obtendo como resultados baixos índices de sintomas de depressão em idosos ativos em suas atividades laborais. Para Magalhães et al. (2016), o trabalho é um organizador social, emocional e financeiro em diversas etapas da vida. Portanto, a aposentadoria pode resultar no sentimento de desvalia nos idosos, aumentando o risco de 
desenvolvimento de sintomas depressivos. Além disso, o envelhecimento é marcado por diversas perdas de familiares, entes queridos e cônjuges. Esses acontecimentos podem expor os idosos ao sentimento de solidão, potencializando o aparecimento de sintomas de depressão, isolamento social, e perda de interesse nas atividades (Ribeiro, Rosa, Sanches, Ribeiro, \& Cassotti, 2017). Ainda, o contexto socioeconômico, como o nível de escolaridade do sujeito, pode estar relacionado a maior predisposição para sintomas depressivos. Desta forma, sugerese que a escolaridade está diretamente relacionada aos recursos adaptativos de enfrentamento e controle do ambiente (Marques et al., 2017).

Em relação ao domínio autonomia, houve uma correlação negativa com a presença de sintomas depressivos. Este construto baseia-se no self do indivíduo, sua capacidade de manter opiniões, independência, e autodeterminação, evitando ceder às pressões sociais (Cachioni et al., 2017; Irigaray, 2009). No questionário aplicado, este domínio é avaliado considerando as seguintes questões "1) Eu tenho coragem de dar minhas opiniões, mesmo que elas sejam contrárias às dos outros”; “9) Eu decido por mim mesmo e por aquilo que acho certo, e não pelas opiniões dos outros" e "15) Eu tenho tendência a ser influenciado (a) por pessoas que tem opiniões fortes".

A autonomia é necessária para as funções cognitivas, pois auxilia o indivíduo a criar sua rotina, manter as atividades de vida diárias e fazer suas escolhas, possibilitando a construção do bem-estar psicológico (Irigaray, 2009). As mudanças biológicas e as doenças crônicas atingem diretamente a autoestima, reduzindo a autonomia, e podem gerar declínios cognitivos que retiram do idoso o controle sobre sua própria vida (Coelho, 2017; Silva, 2017; Tallmann et al., 2013). Desta forma, a presença do domínio autonomia pode favorecer a regulação emocional, ampliando as interações sociais, contribuindo para que o idoso se sinta mais seguro em relação a suas ideias e escolhas (Neri, 2005). O declínio, por sua vez, inicia no processo de envelhecimento, e pode causar sentimento de superproteção do idoso, diminuindo sua independência, participação social, liberdade e individualização (Neri, 2005; Resende \& Neri, 2009; Santos \& Gondim, 2016), o que, por sua vez, pode aumentar o risco de sintomas depressivos.

A literatura converge em relação às perspectivas referentes ao processo de autonomia. Existe a compreensão de que, durante o envelhecimento, há um afastamento de construtos sociais e estigmas, solidificando a independência emocional do sujeito, sua sabedoria para tomada de decisões, e diminuição da suscetibilidade à opinião alheia. Por outro lado, compreende-se que a velhice é um processo individual, não padronizado, no qual cada subjetivo irá remeter a sua experiência de vida, satisfação consigo mesmo e controle do 
ambiente (Caradec, 2013). Portanto, para auxiliar no tratamento da depressão em idosos, é necessário o investimento em atividades físicas, que reduzam o estresse e auxiliem na melhoria da autoestima e autoconfiança. Além disso, possibilitam bem-estar psicológico, pois o idoso será capaz de escolher quais atividades quer fazer, desenvolvendo relações positivas com os outros e aumentando sua interação social, exercitando, assim, seu controle sobre os domínios ambiente e autonomia (Ramos, et al., 2019).

No que se refere ao domínio relações positivas com os outros, os resultados deste estudo reforçam a importância da troca de afeto, convivência e interação social como fator protetivo para o desenvolvimento da depressão em idosos (Maia, Castro, Fonseca, \& Fernández, 2016). Este conceito compreende as relações de satisfação, confiança e proximidade com os outros, indicando que quanto maior as relações positivas menor a sintomatologia depressiva. O estudo de Boff e Gregory, (2019) traz um exemplo disso, indicando a convivência familiar como fator indispensável para o equilíbrio psicológico. Fazem parte deste domínio as questões: “6) Os outros me descrevem como uma pessoa generosa, pronta a compartilhar suas experiências"; "14) É fácil e prazeroso para mim manter relações de proximidade afetiva" e "16) Eu sei que posso confiar em meus amigos e meus amigos sabem que podem confiar em mim”.

O processo de envelhecimento é marcado pela redução de interações sociais, pois há pouca insistência para o desenvolvimento e manutenção de novos vínculos, assim, a relação social estabelecida por meio da interação em espaços de convivência, como grupos de atividades físicas, serviços voluntários, atividades religiosas, etc, torna-se importante (Rabelo \& Neri, 2014). Sabe-se que estas mudanças podem provocar o surgimento de sintomas depressivos, muitas vezes considerado natural na população idosa, caracterizado por perdas sociais, emocionais e econômicas (Barroso, Silva, Neves, \& Braga, 2018; Drago \& Martins, 2011).

Os resultados encontrados em relação ao domínio crescimento pessoal indicam que o envelhecimento saudável depende ativamente do equilíbrio entre potencialidades, força para atingir a autorrealização, vencer obstáculos e abertura a novas experiências. Mediante as dificuldades e limitações, os idosos tendem a passar por situações de estresse, facilitando o sentimento de desânimo, incapacidade e desvalia. Por isso, uma alternativa para preservar este domínio é a possibilidade de exercer trabalhos voluntários, auxiliando na melhoria da autoestima desta população (Teixeira, Nunes, Ribeiro, Arbinaga, \& Vasconcelos-Raposo, 2016; Queroz \& Neri, 2005). 
O construto crescimento pessoal é representado pelas seguintes questões: "4) Eu acho importante ter novas experiências porque elas me fazem pensar sobre mim mesmo e me ajudam a compreender melhor os fatos da vida"; "5) À medida que o tempo passa eu acho que estou cada vez melhor como pessoa" e "8) Eu gosto de vários aspectos da minha personalidade". É fundamental a criação de programas educacionais para fortalecer a participação social na velhice, propiciando empoderamento e segurança, com o intuito de ampliar as capacidades e potencialidades, e diminuir as chances do desenvolvimento de sintomas depressivos em idosos (Coelho, 2017).

Os resultados deste estudo podem ser explicados pelo nível de escolaridade dos participantes (4,59 anos de estudo). Quanto maior o índice de alfabetização, maiores também os recursos adaptativos do sujeito para lidar com atividades estressantes do dia-a-dia, corroborando este achado, um estudo realizado em Minas Gerais com idosos não institucionalizados com média de escolaridade de 3,7 anos, encontrou uma alta sintomatologia depressiva em sua amostra (Ramos, 2015). Da mesma forma, quanto maior a escolaridade maior a pontuação de bem-estar psicológico em idosos (Queroz \& Neri, 2005).

Por fim, essa pesquisa sugere que a atividade física pode auxiliar no bem-estar e na prevenção e proteção de sintomas depressivos em idosos. Este achado corrobora com o estudo de Almeida, Mourão e Coelho (2018), que teve como objetivo verificar o efeito da prática física na cognição, satisfação com a vida e sintomas depressivos em idosos, obtendo como resultado que o exercício físico regular contribui para a saúde mental dos mesmos.

\section{Considerações finais}

Conclui-se que a atividade física pode ser um fator fundamental e protetivo para sintomas de depressão em idosos. A amostra avaliada para a presente pesquisa faz parte de um programa de atividades físicas regulares. O bem-estar pode ser influenciado pelas mudanças causadas pelo processo de envelhecimento (Nakano, Machado, \& Abreu, 2019). Os resultados deste estudo apontam para prevalência significativa de sintomas depressivos na população idosa, impactando diretamente no bem-estar psicológico. A redução do bem-estar devido à depressão pode prejudicar o processo de envelhecimento saudável, uma vez que influencia nos domínios de autonomia, controle do ambiente, relações positivas com os outros e crescimento pessoal. Acredita-se que identificar o nível de bem-estar psicológico pode auxiliar no diagnóstico de depressão no envelhecimento, auxiliando profissionais a criarem estratégias de fortalecimento em domínios que se encontram abaixo do esperado. 
Este estudo apresenta limitações. A literatura utilizada para discutir os dados referentes ao bem-estar psicológico é anterior aos últimos cinco anos, o que pode ter prejudicado a discussão dos dados encontrados. Além disso, a amostra é proveniente de apenas um único local, com predominância de participantes do sexo feminino, o que pode dificultar a generalização dos dados. Sugere-se que sejam desenvolvidos outros estudos que investiguem a relação do bem-estar psicológico e depressão considerando amostras maiores e diversificadas.

A saúde mental e física no envelhecimento está diretamente relacionada à qualidade de vida desta população. Portanto o diagnóstico e tratamento da depressão é um desafio encontrado pelo profissional, devido às diversas comorbidades que acometem os idosos, como doenças, físicas, cognitivas, neurodegenerativas, entre outras. Desta forma, a Psicologia tem papel fundamental no tratamento de doenças psicológicas que afetam a funcionalidade do sujeito, trabalhando juntamente com outros profissionais com o objetivo de proporcionar maior bem-estar psicológico e qualidade de vida nos idosos.

\section{Referências}

Almeida, E., Mourão, I., \& Coelho, E. (2018). Saúde mental em idosos brasileiros: efeitos de diferentes programas de atividade física. Psicologia, Saúde \& Doenças, 19(2), 390404. doi:10.15309/18psd190218

Andrade, A., Duarte, J., Cruz, C., Alburquerque, C., \& Chaves, C. (2019). Inteligência emocional em idosos portugueses. Revista Infad de Psicologia, 4(1), 331-338. doi:10.17060/ijodaep.2019.n1.v4.1566

Barroso, M. L., Silva, S. B. F., Neves, F. P. B., \& Braga, I. B. (2018). A depressão como causa do desenvolvimento de ideação suicida na pessoa idosa e as consequências no âmbito familiar. Revista Multidisciplinar e de Psicologia, 12(41), 66-76. doi:10.14295/idonline.v12i41.1201

Beck, A. T., Steer, R. A., \& Brown, G. K. (1996). Manual for Beck Depression Inventory II. San Antonio. TX: Psychological corporation.

Boff, E., \& Gregory, T. (2019). Avaliação da qualidade de vida em idosos matriculados em um programa universitário para idosos. Revista Ouricuri, 9(1), 27-41. Recuperado de http://www.revistas.uneb.br/index.php/ouricuri/article/view/6039/4161

Cachioni, M., Delfino, L. L., Yassuda, M. S., Batistoni, S. S. T., Melo, R. C., \& Domingues, M. A. R. D. C. (2017). Bem-estar subjetivo e psicológico de idosos participantes de 
Daiane Santos de Oliveira, Manuela Polidoro Lima, Cleber Gibbon Ratto, Tainá Rossi, Rafael Reiman Baptista, Tatiana Quarti Irigaray

uma Universidade Aberta à Terceira Idade. Revista Brasileira de Geriatria e Gerontologia, 20(3), 340-352. doi:10.1590/1981-22562017020.160179

Caradec, V. (2013). Da terceira idade à idade avançada: a conquista da velhice. In M. Goldenberg (Org.), A Bela Velhice (pp. 11-38). Rio de Janeiro, RJ: Record.

Coelho, M. G. (2017). Significados do envelhecimento ativo para idosos: Contribuições da Psicologia. Revista Eletrônica Estácio Papirus, 4(2), 195-214. Retirado de: http://periodicos.estacio.br/index.php/papirussantacatarina/article/view/4276/2105

Cohen, J. (1988). Statistical power analysis for the behavioral sciences. New York, NY: Lawrence Erlbaum Associates Publishers.

Drago, S. M. M. S., \& Martins, R. M. L. (2011). A depressão no idoso (Tese de Doutorado). Instituto Politécnico de Viseu, Viseu, Portugal.

Erikson, E. \& Erikson, J. M. (1998). Life cycle completed: extended version. New York: Norton.

Fave, A. D, Brdar, I., Freire, T., Vella-Brodrick, D., \& Wissing, M. P. (2011). The eudaimonic and hedonic components of happiness: Qualitative and quantitative findings. Social Indicators Research, 100(2), 185-207. doi:10.1007/s11205-010-96325

Gorenstein, C., Pang, W. Y., Argimon, I. L., \& Werlang, B. S. G. (2011). Inventário Beck de Depressão-II. São Paulo, SP: Casa do Psicólogo.

Irigaray, T. Q. (2009). Efeitos de um treino de atenção, memória e funções executivas na cognição, na qualidade de vida e no bem-estar psicológico de idosos saudáveis (Tese de Doutorado em Gerontologia Biomédica). Pontifícia Universidade Católica do Rio Grande do Sul, Porto Alegre, Brasil. Recuperado de http://tede2.pucrs.br/tede2/bitstream/tede/2587/1/411193.pdf

Irigaray, T. Q., Schneider, R. H., \& Gomes, I. (2011). Effects of a cognitive training on the quality of life and well-being of healthy elders. Psicologia: Reflexão e Crítica, 24(4), 810-818. doi:10.1590/S0102-79722011000400022

Kehl, M. R. (2015). O tempo e o cão: A atualidade das depressões. São Paulo, SP: Boitempo Editorial.

Lippert, A. K., Fernandes, F. de. S., Jerônimo, R. N. T., \& Gimes, K. M. (2017). Avaliação de depressão, ansiedade e nível cognitivo em idosos de uma instituição no município de Criciúma, Santa Catarina. Inova Saúde, 6(2), 35-49. doi:10.18616/is.v6i2.3439 
Maia, C. M. L., Castro, F. V., Fonseca, A. M. G., \& Fernández, M. I. R. (2016). Redes de apoio social e de suporte social e envelhecimento ativo. Revista INFAD de Psicología, l(1), 293-306. doi:10.1590/S0034-89102010000600020

Magalhães, J. M., Carvalho, A. M. B., Carvalho, S. M., Alencar, D. C., Moreira, W. C., \& Parente, A. C. M. (2016). Depressão em idosos na estratégia saúde da família: Uma contribuição para a atenção primária. Revista Mineira de Enfermagem, 20(947), 1-6. doi:10.5935/1415-2762.20160016

Marques, J. F. S., Sá, S. C., Filho, W. F., Santos, L. R. S., Prince, K. A., \& Oliveira, M. V. M. (2017). Transtorno depressivo maior em idosos não institucionalizados atendidos em um centro de referência. Arquivos de Ciências da Saúde, 24(4), 20-24. doi:10.17696/2318-3691.24.4.2017.804

Nakano, T. C., Machado, W. L., \& Abreu, I. C. C., (2019). Relações entre estilos de pensar e criar, bem-estar, saúde percebida e estresse na terceira idade. Revista Psico USF, 24(3), 555-568. doi:10.1590/1413-82712019240312

Neri, A. L. (2005). Padrões de envelhecimento/senescência/senilidade (2a ed.). Campinas, SP: Papirus.

Neri, A. L. (1999). Fundamentos para uma escala de geratividade. Campinas, SP: Universidade Estadual de Campinas. Relatório de pesquisa não-publicado.

Queroz, N. C., \& Neri, A. L. (2005). Bem-estar psicológico e inteligência emocional entre homens e mulheres na meia-idade e na velhice. Psicologia: reflexão e crítica, 18(2), 292-299. doi:10.1590/S0102-79722005000200018

Rabelo, D. F., \& Neri, A. L. (2014). A complexidade emocional dos relacionamentos intergeracionais e a saúde mental dos idosos. Pensando famílias, 18(1), 138-153. Recuperado de http://pepsic.bvsalud.org/pdf/penf/v18n1/v18n1a12.pdf

Ramos, F. P., Silva, S. C., Freitas, D. F., Gangussu, L. M. B., Bicalho, A. H., Sousa, B. V. O., . . Guimarães, T. A. (2019). Fatores associados à depressão em idoso. Revista Eletrônica Acervo Saúde, 19(supl.), 1-8. doi:10.25248/reas.e239.2019

Ramos, G. C. F., Carneiro, J. A., Barbosa, A. T. F., Mendonça J. M. G., \& Caldeira, A. P. (2015). Prevalência de sintomas depressivos e fatores associados em idosos no norte de Minas Gerais: Um estudo de base populacional. Jornal Brasileiro de Psiquiatria, 64(2), 122-131. doi:10.1590/0047-2085000000067

Resende, M. C., \& Neri, A. L. (2009). Ajustamento psicológico e perspectiva de velhice pessoal em adultos com deficiência física. Psicologia em estudo, 14(4), 767-776. doi:10.1590/S1413-73722009000400017 
Daiane Santos de Oliveira, Manuela Polidoro Lima, Cleber Gibbon Ratto, Tainá Rossi, Rafael Reiman Baptista, Tatiana Quarti Irigaray

Ribeiro, V. S., Rosa, R. S., Sanches, G. J. C., Ribeiro, I. J. S., \& Cassotti, C. A. (2017). Calidad de vida y depresión en idosos en el contexto domiciliar. Revista Electrónica Enfermería Actual de Costa Rica, 34(1), 1-14. doi:10.15517/REVENF.V0I34.30983

Ryff, C. D. (1989). Happiness is everything, or is it? Explorations on the meaning of psychological well-being. Journal of personality and social psychology, 57(6), 10691081. doi:10.1037/0022-3514.57.6.1069

Ryff, C. D. (2014). Psychological well-being revisited: advances in the science and practice of eudaimonia. Psychotherapy and psychosomatics, 83(1), 10-28. doi:10.1159/000353263

Ryff, C. D., \& Keyes, C. L. M. (1995). The structure of psychological well-being revisited. Journal of personality and social psychology, 69(4), 719. doi:10.1037/00223514.69.4.719

Ryff, C. D., \& Singer, B. H. (2008). Know thyself and become what you are: A eudaimonic approach to psychological well-being. Journal of happiness studies, 9(1), 13-39. doi:10.1007/s10902-006-9019-0

Santos, S. V., \& Gondim, I. J. S. (2016). Regulação emocional, bem-estar psicológico e bemestar subjetivo. Estudos de Psicologia (Natal), 21(1), 58-68. doi:10.5935/16784669.20160007

Silva, A. R., Sgnaolin, V., Nogueira, E. L., Loureiro, F., Engroff, P., \& Gomes, I. (2017). Doenças crônicas não transmissíveis e fatores sociodemográficos associados a sintomas de depressão em idosos. Jornal Brasileiro de Psiquiatria, 66(1), 45-51. doi:10.1590/0047-2085000000149

Silva, J. S. V. (2010). Funcionalidade e bem-estar psicológico em idosos residentes na comunidade: Um estudo exploratório (Dissertação de Mestrado). Universidade de Lisboa, Lisboa, Portugal. Recuperado de https://repositorio.ul.pt/bitstream/10451/2827/1/ulfp037541_tm.pdf

Silva, S. H. (2017). Qualidade de vida, depressão e capacidade laboral dos idosos trabalhadores de um hospital de clínicas (Dissertação de Mestrado). Universidade Federal do Triângulo Mineiro, Uberaba, Minas Gerais. Recuperado de http://bdtd.uftm.edu.br/bitstream/tede/543/5/Dissert\%20Sheron\%20H\%20Silva.pdf

Silva, M. A. D., Wendt, G. W., \& Argimon, I. I. D. L. (2018). Inventário de depressão de beck II: análises pela teoria do traço latente. Avaliação Psicológica, 17(3), 339-350. doi:10.15689/ap.2018.1703.14651.07 
Tallmann, A. E. C., Lenardt, M. H., Kletemberg, D. F., Michel, T., \& Lourenço, T. M. (2013). Envelhecimento e bem-estar psicológico: Uma revisão integrativa. Ciência Cuidado e Saúde, 12(3), 599-605. doi:10.4025/cienccuidsaude.v12i3.15949

Teixeira, C. M., Nunes, F. M. S., Ribeiro, F. M. S., Arbinaga, F., \& Vasconcelos-Raposo, J. (2016). Atividade física, autoestima e depressão em idosos. Cuadernos de psicologia del deporte, 16(3), 55-66. Recuperado de https://revistas.um.es/cpd/article/view/278431

Tsai, J. C., Chen, C. W., Chu, H., Yang, H. L., Chung, M. H., Liao, Y. M., \& Chou, K. R. (2016). Comparing the sensitivity, specificity, and predictive values of the Montreal Cognitive Assessment and Mini-Mental State Examination when screening people for mild cognitive impairment and dementia in Chinese population. Archives of psychiatric nursing, 30(4), 486-491. doi:10.1016/j.apnu.2016.01.015

Warr, P. (2011). Work, happiness, and unhappiness. Reino Unido: Psychology Press. doi:10.4324/9780203936856

\section{Endereço para correspondência}

\section{Daiane Santos de Oliveira}

Avenida Ipiranga, 6681, P.11, sala 939, Partenon, Porto Alegre - RS, Brasil. CEP 90619-900

Endereço eletrônico: daianehausen@gmail.com

\section{Manuela Polidoro Lima}

Avenida Ipiranga, 6681, P.11, sala 939, Partenon, Porto Alegre - RS, Brasil. CEP 90619-900

Endereço eletrônico: manuela.lima@acad.pucrs.br

\section{Cleber Gibbon Ratto}

Rua Pedro Joaquim Salgado, 80, Rio Branco, Porto Alegre - RS, Brasil. CEP 90420-060

Endereço eletrônico: rattocleber@gmail.com

\section{Tainá Rossi}

Avenida Ipiranga, 6681, P.11, sala 939, Partenon, Porto Alegre - RS, Brasil. CEP 90619-900

Endereço eletrônico: tainabrossi@gmail.com

\section{Rafael Reiman Baptista}

Avenida Ipiranga, 6681, P.11, sala 939, Partenon, Porto Alegre - RS, Brasil. CEP 90619-900

Endereço eletrônico: rafael.baptista@pucrs.br

\section{Tatiana Quarti Irigaray}

Avenida Ipiranga, 6681, P.11, sala 939, Partenon, Porto Alegre - RS, Brasil. CEP 90619-900

Endereço eletrônico: tatiana.irigaray@pucrs.br 
Recebido em: 03/06/2018

Reformulado em: 19/08/2019

Aceito em: 24/10/2019

\section{Notas}

* Psicóloga - Centro Universitário Metodista (IPA). Colaboradora no grupo de pesquisa ARIHA da PUCRS.

** Psicóloga, mestre em Ciências da Saúde pelo Programa de Pós-Graduação do Hospital de Câncer de Barretos e doutora em Psicologia pela PUCRS.

*** Psicólogo e Doutor em educação pela PUCRS, coordenador do grupo de pesquisa Cultura Contemporânea, Sociabilidades e Praticas Educativas do IPA.

**** Psicóloga pela Faculdade Meridional (IMED). Colaboradora do grupo de pesquisa ARIHA da PUCRS.

***** Educador Físico (ULBRA). Mestre em fisiologia do exercício e Doutor em Biomecânica pela Universidade Federal do Rio Grande do Sul.

****** Psicóloga (UFRGS). Pós-Doutorado em Psicologia pela PUCRS. Coordenadora do grupo Avaliação, Reabilitação e Interação Humano-Animal (ARIHA).

Este artigo de revista Estudos e Pesquisas em Psicologia é licenciado sob uma Licença Creative Commons Atribuição-Não Comercial 3.0 Não Adaptada. 